\title{
Tunable Master-Oscillator Power-Amplifier Based on Chirped Quantum-Dot Structures
}

\author{
Y. Ding, A. Alhazime, D. Nikitichev, K. Fedorova, M. Ruiz, M. Tran, Y. Robert, A. Kapsalis, H. Simos, \\ C. Mesaritakis, T. Xu, P. Bardella, M. Rossetti, I. Krestnikov, D. Livshits, I. Montrosset, D. Syvridis, \\ M. A. Cataluna, M. Krakowski, and E. Rafailov
}

\begin{abstract}
A broadly tunable master-oscillator power-amplifier (MOPA) picosecond optical pulse source is demonstrated, consisting of an external-cavity passively mode-locked laser diode with a tapered semiconductor amplifier. By employing chirped quantum-dot structures on both the oscillator's gain chip and amplifier, a wide tunability range between $1187 \mathrm{~nm}$ and $1283 \mathrm{~nm}$ is achieved. Under mode-locked operation, the highest output peak power of $4.39 \mathrm{~W}$ is achieved from the MOPA, corresponding to a peak power spectral density of $31.4 \mathrm{dBm} / \mathrm{nm}$.
\end{abstract}

Index Terms--Chirped quantum dots, master-oscillator poweramplifier, mode-locking, tunability.

\section{INTRODUCTION}

$\mathbf{O}$ uantum-dot external-cavity passively mode-locked lasers (QD-ECMLLs) are excellent candidates for versatile ultrashort pulse generation due to quantum-dot (QD) lasers' inherent merits of low threshold current density, low optical losses and low noise characteristics [1-4], as well as the flexibility that external-cavity mode-locking configurations can offer such as a broad tunability for both the pulse repetition rate [5-7] and the wavelength [8]. The potential for wavelength tunability in InAs/GaAs QD modelocked lasers has been demonstrated in grating-coupled QDECMLLs [8] or through dual-mode optical injection [9].

Manuscript received May 09, 2012. This work was funded within the Seventh Framework Program "FAST-DOT", through Grant No. 224338. Y. Ding acknowledges financial support from a Marie Curie Fellowship. M. A. Cataluna acknowledges also financial support through a Royal Academy of Engineering/EPSRC Research Fellowship.

Y. Ding, A. Alhazime, D. Nikitichev, K. Fedorova, M. A. Cataluna, and E. Rafailov are with School of Engineering, Physics and Mathematics,

University of Dundee, Dundee DD1 4HN, UK (email: y.ding@dundee.ac.uk; A.Alhazime@dundee.ac.uk; D.Nikitichev@dundee.ac.uk;

k.a.fedorova@dundee.ac.uk; M.A.Cataluna@dundee.ac.uk;

E.U.Rafailov@dundee.ac.uk).

M. Ruiz, M. Tran, Y. Robert, and M. Krakowski are with III-V Lab, 1 Av Augustin Fresnel, Campus de Polytechnique, 91767 Palaiseau, France (email: myke.ruiz@3-5lab.fr; michael.tran@3-5lab.fr; yannick.robert@3-5lab.fr; michel.krakowski@3-5lab.fr).

T. Xu, P. Bardella, M. Rossetti, and I. Montrosset are with Dipartimento di Elettronica e Telecomunicazioni, Politecnico di Torino, I-10129 Turin, Italy (email: Tianhong.Xu@polito.it; paolo.bardella@polito.it; mattia.rossetti@polito.it; ivo.montrosset@polito.it).

A. Kapsalis, H. Simos, C. Mesaritakis, and D. Syvridis are with National and Kapodistrian University of Athens, Panepistimiopolis, Ilissia, Athens 15784, Greece (email: alex@di.uoa.gr; simos@di.uoa.gr; cmesar@di.uoa.gr; dsyvridi@di.uoa.gr).

I. Krestnikov and D. Livshits are with Innolume GmbH, KonradAdenauer-Allee 11, 44263 Dortmund, Germany (email: Igor.Krestnikov@innolume.com; daniil.livshits@innolume.com).
From these prior works, the widest tunability range was demonstrated in [8], where the gain chip used in the external cavity was based on a QD structure which consisted of 10 identical InAs/GaAs QD layers. Owing to the inherent broad gain and absorption bandwidth afforded by QD structures, it was already possible to achieve tunable picosecond operation, albeit only within two separate ranges: between $1170 \mathrm{~nm}-$ $1220 \mathrm{~nm}$ across the excited-state (ES) transition, and in the range of $1265 \mathrm{~nm}-1295 \mathrm{~nm}$, corresponding to the ground-state (GS) transition. A narrow-ridge QD semiconductor optical amplifier (SOA) was used to boost the power for diagnostics, but no information was provided on the resulting output power [8]. The tunability performance can be significantly enhanced by the use of non-identical or chirped QD structures, whereby the growth of different groups of QD layers is engineered so that each group displays a different central emission wavelength, allowing for a broadband spectral coverage between the GS and ES transitions of the different groups [10]. The merit of using such chirped QD structures has been previously demonstrated in widely-tunable QD external-cavity lasers in CW operation [11-12]. In this paper we demonstrate that the use of nonidentical or chirped QD structures on both oscillator and tapered amplifier enables the generation of high-power picosecond pulses continuously tunable between $1187 \mathrm{~nm}$ and $1283 \mathrm{~nm}$. This is a spectral region of great interest for various applications, which have been addressed by the use of optical parametric oscillators, Raman lasers or Bismuth-doped fiber lasers. For example, some modalities of multi-photon imaging benefit from this spectral band, due to its deep penetration depth into biological tissues. It is also of significant relevance for second-harmonic generation into the yellow-orange spectral range, which is in great demand for a number of biomedical applications and ophthalmology in particular. The picosecond MOPA system presented in this paper represents an attractive light source for these applications, with its lower cost, smaller footprint and higher wall-plug efficiency than currently available sources in this spectral region.

\section{EXPERIMENTAL SETUP}

The scheme of the experimental setup is shown in Fig. 1. The tunable MOPA system consists of a QD-ECMLL and a tilted and tapered QD-SOA. The QD-ECMLL is based on a two-section gain chip and a diffraction grating (DG) with 600 grooves $/ \mathrm{mm}$ and blaze wavelength at $1.25 \mu \mathrm{m}$. Both gain chip and tapered SOA were fabricated from the same wafer, grown 


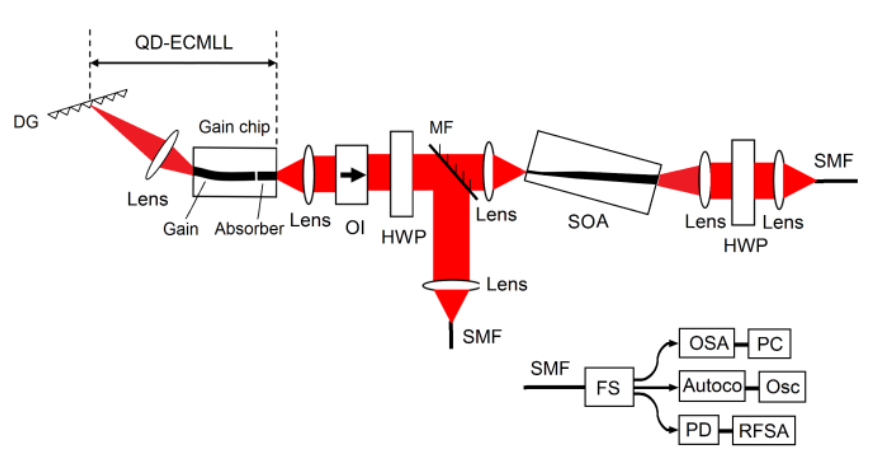

Fig. 1. Configuration of the tunable MOPA system and characterization setup. DG: diffraction grating; OI: optical isolator; HWP: half wave plate; MF: mirror flipper; SMF: single-mode fiber; FS: fiber splitter; OSA: optical spectrum analyzer; Autoco: autocorrelator; Osc: oscilloscope; PD: photo detector; RFSA: RF spectrum analyzer.

by Innolume on a $\mathrm{n}^{+}$-GaAs (100) substrate by molecular beam epitaxy (MBE). Its active region contained ten layers of chirped InAs/GaAs QDs in order to achieve broad gain spectrum, using an epitaxy similar to that used in previous papers [10-11], whereby the 10 QD layers in the active region were made up of 3 groups with different emission peak positions at $1211 \mathrm{~nm}, 1243 \mathrm{~nm}$ and $1285 \mathrm{~nm}$. In order to achieve these various shifted spectral emissions, the InGaAs capping layer thickness was slightly different between the three groups, which resulted in an overall variation of the average size of the quantum dots, for each group of dots. As a result, there are two main contributions to the inhomogeneous broadening: the first contribution is linked with the statistical fluctuation of size of the QDs within each group of QD layers and which is inherent to their self-assembled growth; the second contribution results from the intentional variation in capping layer thickness which changes the average size of the dots among the three different groups of QD layers. Moreover, each group of QD layers allows access to their ground and excited-state optical transitions, which are then slightly shifted from group to group. This approach allows for a continuous wavelength tuning between the ground and excited-state optical transitions of the different QD groups. The two-section gain chip used for the QD-ECMLL consists of a gain section and a saturable absorber (SA) section. The 6$\mu \mathrm{m}$-wide ridge waveguide in the gain section is bent and terminated at an angle of $7^{\circ}$ relative to the cleaved facet, in combination with an antireflection (AR) coating $\left(\mathrm{R} \sim 10^{-5}\right)$, while the front facet was coated with a $\mathrm{R} \sim 1 \%$ coating, which allowed for cavity feedback and a high output power. The total chip length is $4 \mathrm{~mm}$, with an 800- $\mu \mathrm{m}$-long SA section placed near the front facet. The DG reflected the second order diffraction beam back to the gain chip to achieve wavelength tunability. We selected the second-order diffraction from the 600 grooves $/ \mathrm{mm}$ grating because the optical spectra width achieved under this second-order is much narrower than that obtained with the first-order configuration. This strategy was followed in order to get a higher power spectral density, with a view to use this MOPA system in second-harmonic generation applications, where the necessary phase-matching conditions restrict the useable spectral bandwidth of the fundamental radiation. Furthermore, although the pulse duration from the second-order diffraction is slightly broader than that from the first-order diffraction under the similar conditions, the difference is not noteworthy, and as such, the peak power was practically the same in both configurations. A fundamental repetition frequency of $\sim 1.3 \mathrm{GHz}$ was primarily set up by adjusting the external cavity length. The output beam from the gain chip was coupled onto the front facet of broadband QD-SOA after optical isolation and polarization control. The length of the SOA is $6 \mathrm{~mm}$. The width of the gain-guided waveguide of the SOA changed from $14 \mu \mathrm{m}$ at the input facet to $80 \mu \mathrm{m}$ at the output facet. Both facets of SOAs were AR-coated, in combination with a tilt waveguide resulting in a residual reflectivity of $\sim 10^{-5}$. The tapered SOAs were fabricated by III-V Lab according to University of Athens and Politecnico di Torino's design and simulation. Details on these devices are the subject of a different work and will be published elsewhere. Unless stated otherwise, both chips were operated at $20^{\circ} \mathrm{C}$.

\section{RESULTS}

For the tunable MOPA in mode-locked operation, Fig. 2(a) shows the optical spectra obtained for a 600-mA bias current applied to the gain chip (corresponding to $3.1 \mathrm{kA} / \mathrm{cm}^{2}$ ), and SOA current of $2185 \mathrm{~mA}\left(710 \mathrm{~A} / \mathrm{cm}^{2}\right)$. The reverse bias applied on the gain-chip's saturable absorber was optimized between 0-6 $\mathrm{V}$ to achieve the shortest pulse duration for each wavelength (as shown in Fig. 2b). A strong ASE feature can be seen for the 1274-nm laser amplification (Fig. 2(a)), which is due to a large detuning between the laser wavelength and SOA gain maximum under the abovementioned operation conditions. The peak power and gain achieved from the SOA (after subtracting the ASE power) as well as the corresponding pulse duration are represented in Fig. 2(b). The achieved gain is significantly higher on the blue side of the spectral range. Given the high level of current injection in the tapered SOA, this trend can be accounted for due to the saturation across the GS transitions and stronger carrier filling of the higher-energy ES levels - which also have a higher degeneracy and as such, enable a higher level of gain on the blue side of the amplification spectral range.

The peak power spectral density at various wavelengths is depicted in Fig. 2 (c). The highest peak power of $4.39 \mathrm{~W}$ is achieved at the wavelength of $1226 \mathrm{~nm}$, which corresponds to a peak power spectral density of $31.4 \mathrm{dBm} / \mathrm{nm}$.

In Fig. 3, the pulse and RF characteristics for two representative operation wavelengths of $1226 \mathrm{~nm}$ and 1214 $\mathrm{nm}$ are presented. The high signal-to-noise ratio as well as the large number of harmonics in the RF spectra indicates the high quality of mode-locking, as shown in Fig. 3 (b), (d). The fundamental repetition rate changed slightly from $1.316 \mathrm{GHz}$ to $1.324 \mathrm{GHz}$ for the two different wavelengths due to variations in the refractive index of the (gain chip) semicond- 

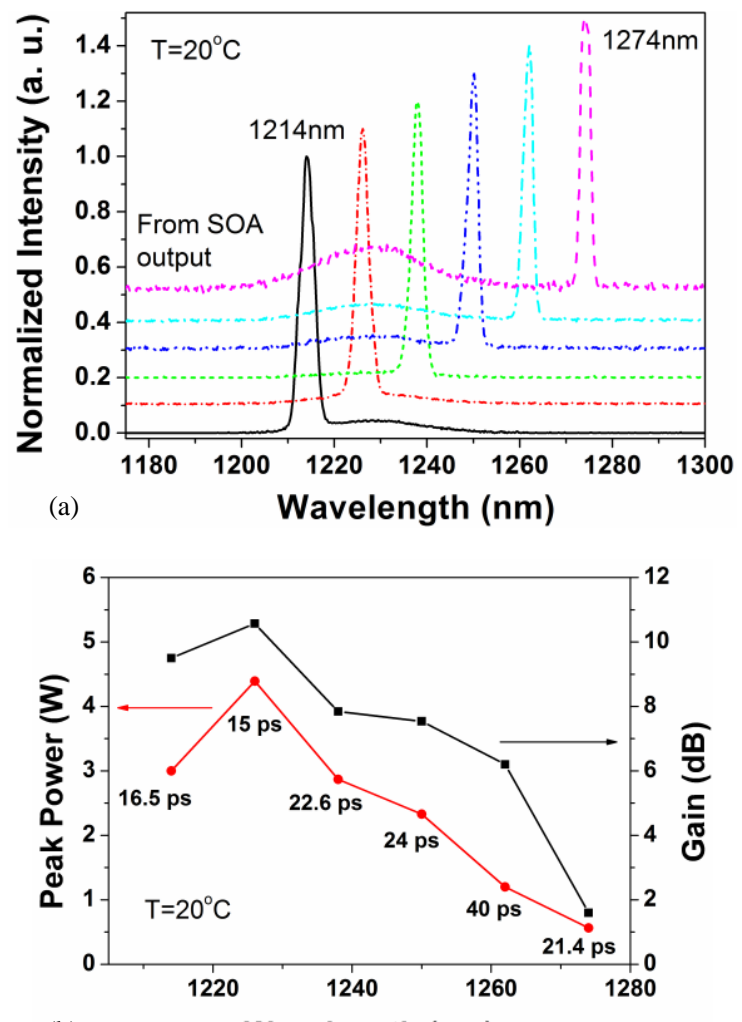

(b)

Wavelength $(\mathrm{nm})$

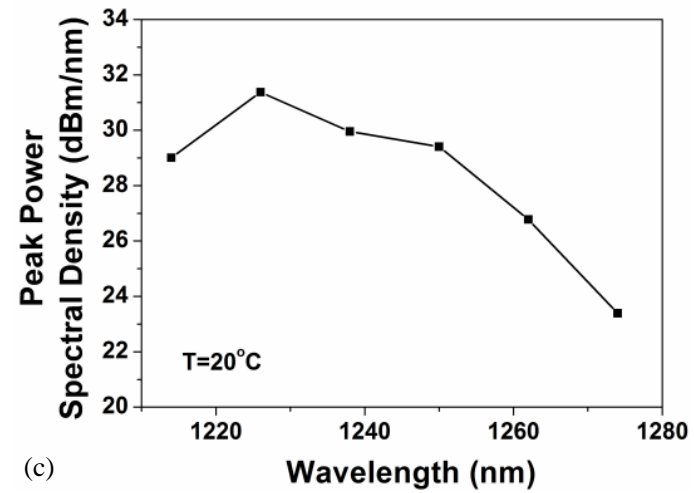

Fig. 2. (a) Optical spectra of tunable MOPA in mode-locked operation with gain chip current of $600 \mathrm{~mA}$, reverse bias of 0-6 V, and SOA current of 2185 $\mathrm{mA}\left(710 \mathrm{~A} / \mathrm{cm}^{2}\right)$. (b) Output peak power and gain from SOA at different wavelengths. (c) Peak power spectral density from SOA vs. wavelength.

uctor, which stems from a combination of spectrallydependent refractive index change along with the variation of effective overall refractive index with the different reverse bias applied to the saturable absorber.

Importantly, it is possible to achieve a wider tunability range in mode-locked operation by increasing the bias current applied to the gain chip, as shown in Fig. 4. This widening of the tunability range occurs preferentially on the blue side of the spectrum, which can be attributed to the increasingly stronger carrier filling of the higher-energy, higherdegeneracy ES levels, as previously observed also in CW tunable QD lasers [11]. Under the gain chip current of
$900 \mathrm{~mA}$, a 96-nm tuning range was therefore achieved with stable fundamental mode-locked operation. It is important to add in this context that the peak power achieved from the QDECMLL oscillator was relatively constant with increasing gain chip current due to the concurrent pulse broadening and increase of average power, as previously shown also in [3]. Our investigation also shows that a $\sim 20 \%$ power increase can be attained at $10{ }^{\circ} \mathrm{C}$ with an SOA current of $2 \mathrm{~A}$, which means that a higher peak power from the SOA output can be easily achieved under a lower SOA operation temperature. Finally, and if the target application demands it, a lower pulse repetition rate should be achievable with this QD-ECMLL oscillator, according to our previous work (undertaken at a fixed wavelength of $1.27 \mu \mathrm{m}$ ), in which a $191-\mathrm{MHz}$ repetition rate was achieved [5].
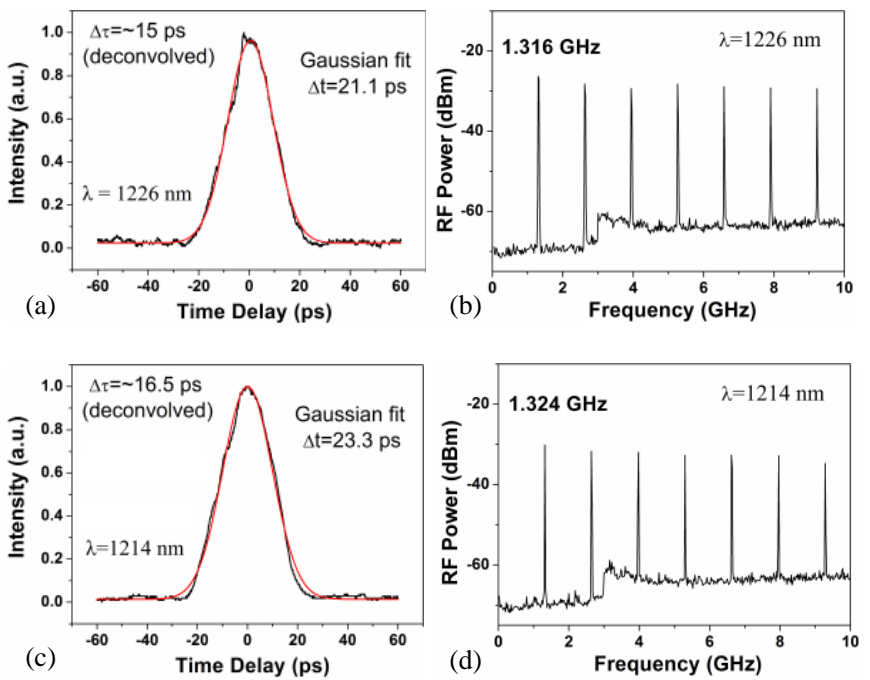

Fig. 3. (a), (b) Autocorrelation trace and RF spectrum at reverse bias of $5 \mathrm{~V}$ and forward current of $600 \mathrm{~mA}$ on the gain chip and SOA current of 2185 $\mathrm{mA}$ at $20{ }^{\circ} \mathrm{C}$ at the operation wavelength of $1226 \mathrm{~nm}$. (c),(d) Counterpart for operation at $1214 \mathrm{~nm}$, with a reverse bias of $2 \mathrm{~V}$.

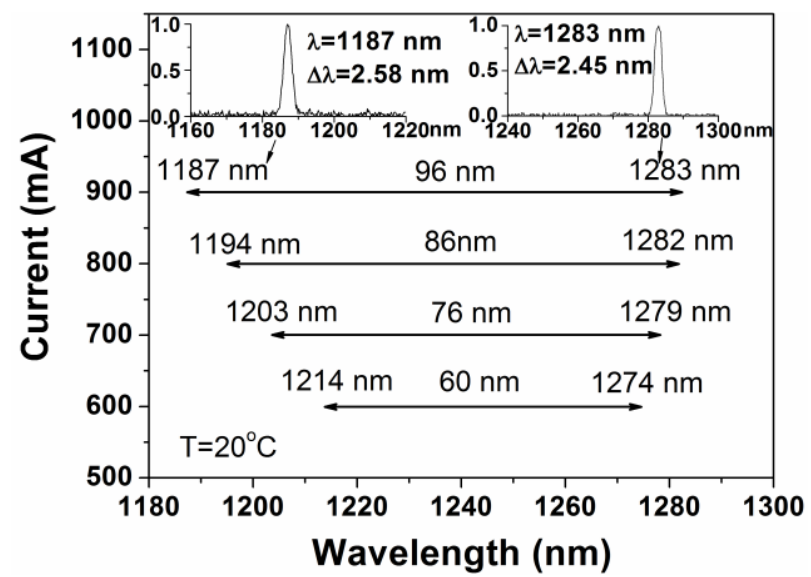

Fig. 4. Mode-locked tuning range limits from the gain chip or the MOPA under different forward currents applied on the gain chip at $20^{\circ} \mathrm{C}$. Insets: two exemplary optical spectra of the mode-locked pulses at the limit positions. 
The present QD-ECMLL was designed for high average power and peak power operation, thus corresponding to a high figure-of-merit (FOM), defined as the product of the average power and the peak power $\left(\mathrm{P}_{\mathrm{avg}} \times \mathrm{P}_{\text {peak }}\right)$. Such FOM reflects the suitability of the laser source for nonlinear excitation (as for example, in multi-photon imaging [13]). It's also worth mentioning that the broad wavelength tunability was not available from the MOPA system based on the unchirped (identical) QD structures in our previous study [13]. Shorter pulse durations could also be obtained in the future by a number of approaches such as increasing the value of AR coating on the front facet, increasing the absorber-to-totallength ratio, or employing a intra-cavity or extra-cavity pulse compression setup. In adopting these approaches, consideration will be given whether the resulting FOM can be maintained or preferably enhanced, with a view to maximize the applicability of this laser source.

\section{CONCLUSION}

An all-semiconductor tunable MOPA picosecond optical pulse source with a tunability range between $1187 \mathrm{~nm}$ and $1283 \mathrm{~nm}$ was demonstrated. Such performance was achieved by using chirped QD structures in both oscillator and tapered amplifier. The highest peak power of $4.39 \mathrm{~W}$ is achieved, which corresponds to a peak power spectral density of 31.4 $\mathrm{dBm} / \mathrm{nm}(1.38 \mathrm{~W} / \mathrm{nm})$. This MOPA system represents a versatile, compact and low-cost source, well-suited for efficient and tunable second-harmonic generation applications into the yellow-orange spectral regions.

\section{RFFERENCES}

X. D. Huang, A. Stintz, H. Li, L. F. Lester, J. Cheng, and K. J. Malloy, "Passive mode-locking in $1.3 \mu \mathrm{m}$ two-section InAs quantum dot lasers," Applied Physics Letters, vol. 78, pp. 28252827, May 2001.

[2] E. U. Rafailov, M. A. Cataluna, and W. Sibbett, "Mode-locked quantum-dot lasers," Nat. Photonics vol. 1, pp. 395-401, Jul 2007.

[3] M. A. Cataluna, Y. Ding, D. I. Nikitichev, K. A. Fedorova, and E. U. Rafailov, "High-Power Versatile Picosecond Pulse Generation from Mode-Locked Quantum-Dot Laser Diodes," IEEE Journal of Selected Topics in Quantum Electronics, vol. 17, pp. 1302-1310, Sep-Oct 2011.
[4] M. G. Thompson, A. R. Rae, X. Mo, R. V. Penty, and I. H. White, "InGaAs Quantum-Dot Mode-Locked Laser Diodes," IEEE J. Sel. Top. Quantum Electron., vol. 15, pp. 661-672, 2009.

[5] Y. Ding, M. A. Cataluna, D. Nikitichev, I. Krestnikov, D. Livshits, and E. Rafailov, "Broad Repetition-Rate Tunable Quantum-Dot External-Cavity Passively Mode-Locked Laser with Extremely Narrow Radio Frequency Linewidth," Applied Physics Express, vol. 4, p. 062703, Jun 2011.

[6] Y. Ding, D. I. Nikitichev, I. Krestnikov, D. Livshits, M. A. Cataluna, and E. U. Rafailov, "Fundamental and harmonic modelocking with pulse repetition rate between $200 \mathrm{MHz}$ and $6.8 \mathrm{GHz}$ in a quantum-dot external-cavity laser " Lasers and Electro-Optics Europe (CLEO EUROPE/EQEC), 2011 Conference on and 12th European Quantum Electronics Conference, Munich, Germany, 2011.

[7] M. Xia, M. G. Thompson, R. V. Penty, and I. H. White, "ExternalCavity Mode-Locked Quantum-Dot Laser Diodes for Low Repetition Rate, Sub-Picosecond Pulse Generation," IEEE Journal of Selected Topics in Quantum Electronics, vol. 17, pp. 1264-1271, Sep-Oct 2011.

[8] J. Kim, M. T. Choi, W. Lee, and P. J. Delfyett, "Wavelength tunable mode-locked quantum-dot laser - art. no. 62430M," in Enabling Photonics Technologies for Defense, Security, and Aerospace Applications II. vol. 6243, M. J. Hayduk, A. R. Pirich, E. J. Donkor, and P. J. Delfyett, Eds., ed Bellingham: SPIE-Int Soc Optical Engineering, 2006, pp. M2430-M2430.

[9] T. Habruseva, S. O'Donoghue, N. Rebrova, D. A. Reid, L. P. Barry, D. Rachinskii, G. Huyet, and S. P. Hegarty, "Quantum-Dot Mode-Locked Lasers With Dual-Mode Optical Injection," IEEE Photonics Technology Letters, vol. 22, pp. 359-361, Mar 2010.

[10] A. Kovsh, I. Krestnikov, D. Livshits, S. Mikhrin, J. Weimert, and A. Zhukov, "Quantum dot laser with $75 \mathrm{~nm}$ broad spectrum of emission," Optics Letters, vol. 32, pp. 793-795, Apr 2007.

[11] K. A. Fedorova, M. A. Cataluna, I. Krestnikov, D. Livshits, and E. U. Rafailov, "Broadly tunable high-power InAs/GaAs quantumdot external cavity diode lasers," Optics Express, vol. 18, pp. 19438-19443, Aug 2010.

[12] A. Y. Nevsky, U. Bressel, I. Ernsting, C. Eisele, M. Okhapkin, S. Schiller, A. Gubenko, D. Livshits, S. Mikhrin, I. Krestnikov, and A. Kovsh, "A narrow-line-width external cavity quantum dot laser for high-resolution spectroscopy in the near-infrared and yellow spectral ranges," Applied Physics B-Lasers and Optics, vol. 92, pp. 501-507, Sep 2008.

Y. Ding, R. Aviles-Espinosa, M. A. Cataluna, D. Nikitichev, M. Ruiz, and Y. R. M. Tran, A. Kapsalis, H. Simos, C. Mesaritakis, T. $\mathrm{Xu}$, P. Bardella, M. Rossetti, I. Krestnikov, D. Livshits, Ivo Montrosset, D. Syvridis, M. Krakowski, P. Loza-Alvarez, and E. Rafailov, "High peak-power picosecond pulse generation at 1.26 $\mu \mathrm{m}$ using a quantum-dot-based external-cavity mode-locked laser and tapered optical amplifier," Optics Express, vol. 20, pp. 1430814320, 2012. 